\title{
Advancements in X-Ray Analysis for Correlative Microscopy
}

Jeff Gelb ${ }^{1}$, Sylvia Lewis ${ }^{1}$, SH Lau ${ }^{1}$, Janos Kirz ${ }^{1,2}$, and Wenbing Yun ${ }^{1}$

${ }^{1 .}$ Sigray, Inc., Concord, CA, USA

2. Lawrence Berkeley National Laboratory, Berkeley, CA, USA

Modern microscopy laboratories have, in recent years, witnessed a growth in adoption of X-ray technologies. X-ray techniques offer exceptionally high penetrating powers as compared to conventional electron- and optical microscopy approaches, providing information about a material's surface and subsurface with minimal specimen preparation [1]. While these techniques often do not match the spatial resolution capabilities of conventional characterization approaches, such as SEM and TEM, the unique advantages of X-ray techniques for non-destructive characterization have made them a popular choice for correlative microscopy workflows, as an informative step on the way to higher-resolution imaging [2].

In spite of the intrinsic advantages of X-ray imaging and spectroscopy, the techniques themselves have encountered several challenges within the framework of a central laboratory. Conventional laboratory $\mathrm{X}$-ray sources suffer from relatively low brightnesses, which can lead to long acquisition times, often requiring multiple days for high-quality results. X-ray absorption spectroscopy, in particular, has fallen out of favor in the laboratory, simply due to very long exposure times of days or longer for a single study [3]. X-ray fluorescence spectrometers, similarly, couple detection sensitivities to source brightnesses and, in turn, source spot sizes, which means that conventional instruments struggle to detect trace elements of less than the parts-per-million ( $\mathrm{ppm}$ ) in a realistic time scale. Furthermore, since suppliers for imaging instrumentation often do not provide spectroscopic analyzers, and vice-versa, pulling together the data from multiple instruments produced by multiple vendors creates new challenges in big data handling, thus complicating workflows in correlative microscopy [4].

In our work, we have created a novel suite of X-ray instrumentation, featuring a state-of-the-art laboratory X-ray source, ultra-high performance X-ray optics, and a unified data structure, which alleviates many of the challenges in X-ray characterization, particularly for correlative microscopy workflows. The X-ray source uses a fine array of micro targets embedded in diamond, which provides excellent heat dissipation and X-ray source brightnesses $\sim 50 \mathrm{x}$ those of comparable laboratory setups. Target arrays may be customized with different materials, allowing the end user to select the most appropriate energy (or wavelength) for providing optimal results. These fine-array, structured targets are then paired with specially-designed, parabolic capillary lenses, which optimize the usage of the X-ray source and reduce irregularities in the illumination profile across the specimen. Through the additional integration of crystal monochromators and/or Fresnel zone plate diffractive lenses, these different components have been configured for probing microstructure/ultrastructure with down to $40 \mathrm{~nm}$ resolution (e.g., XRM), elemental composition with parts-per-billion (ppb) detection sensitivities (e.g., $\mu \mathrm{XRF}$ ), and chemical state analysis in the minutes-to-hours regime (e.g., XAS). These integrated systems then produce data in a common format, following the international research standard Data Exchange convention of HDF5 [4], thus simplifying downstream data processing workflows as users move on to the next step in their experiment(s). 
References:

[1] A P Merkle and J Gelb, Microscopy Today 21 (2013), p. 10-15.

[2] T L Burnett, S A McDonald, A Gholinia, R Geurts, M Janus, T Slater, SJ Haigh, C Ornek, F Almuaili, D L Engelberg, G E Thompson, and P J Withers, Scientific Reports 4 (2014), p. 1-6.

[3] GT Seidler, DR Mortensen, AJ Remesnik, JI Pacold, NA Ball, N Barry, M Styczinski, OR Hoidn, Review of Scientific Instruments 85 (2014).

[4] F De Carlo, D Gürsoy, F Marone, M Rivers, D Y Parkinson, F Khan, N Schwarz, D J Vine, S Vogt, S-C Gleber, S Narayanan, M Newville, T Lanzirotti, Y Sun, Y P Hong and C Jacobsen, Journal of Synchrotron Radiation 21 (2014), p. 1224-1230.
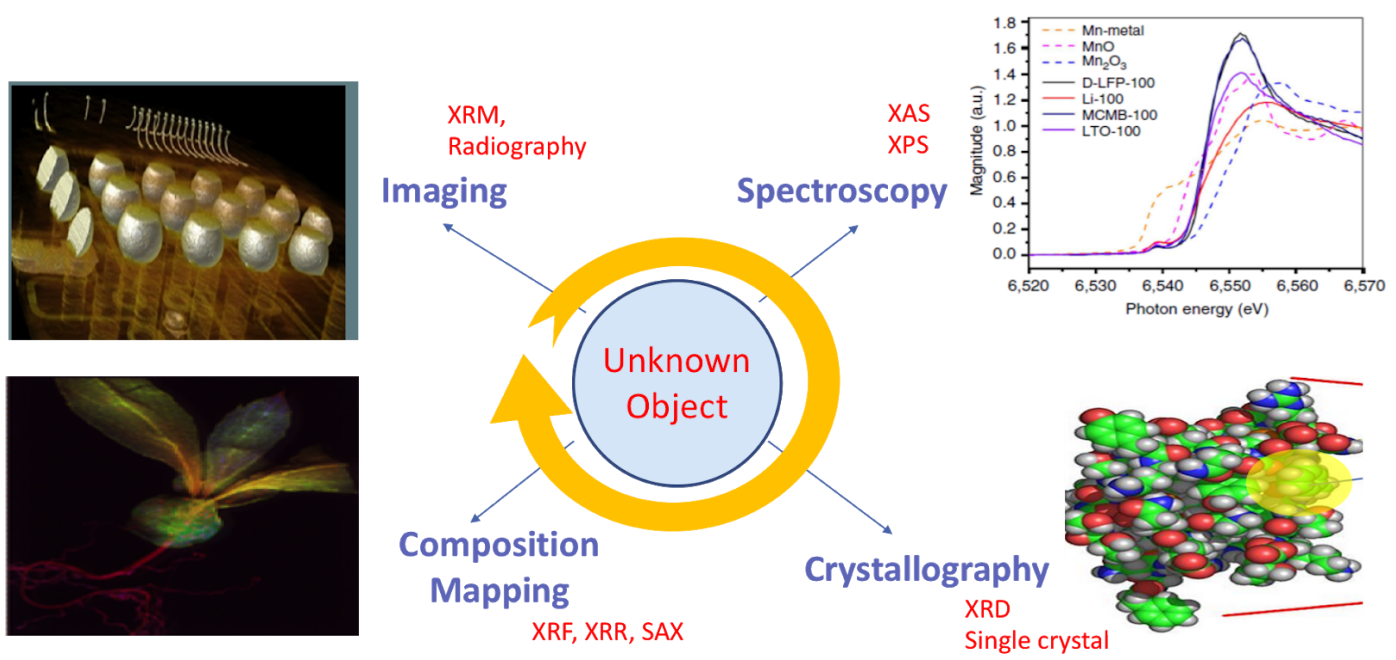

Figure 1. Novel X-ray instrumentation serves many needs, including those of structural investigations (imaging), compositional analysis, and chemical state determination (spectroscopy), as well as crystallography.

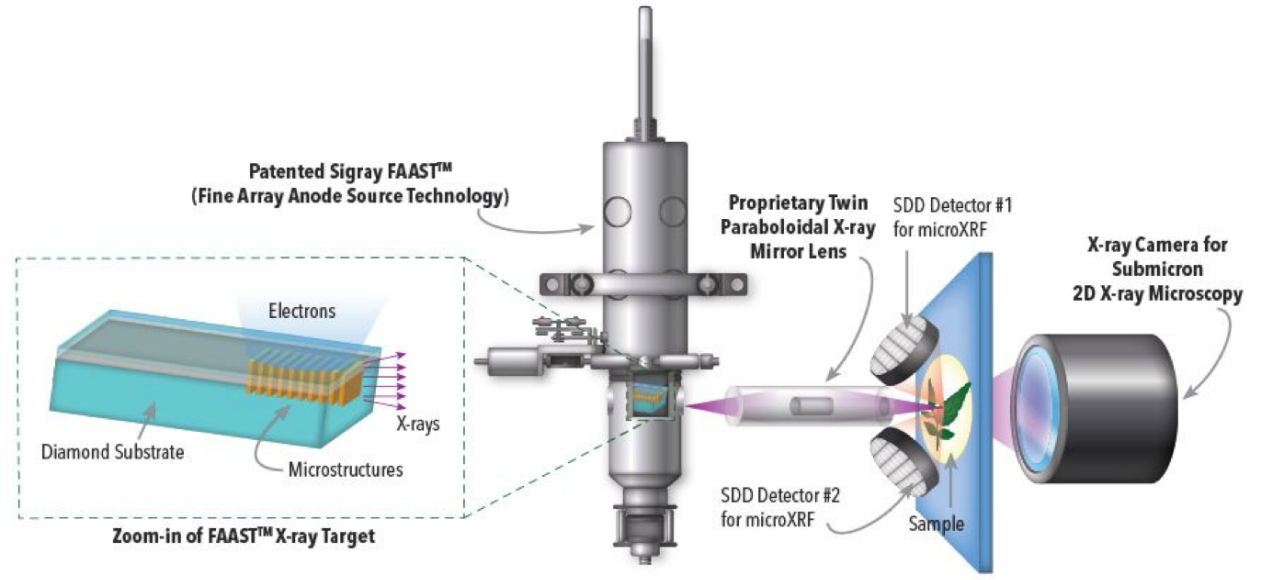

Figure 2. The micro X-ray fluorescence spectrometer (micro-XRF) uses a combination of the novel X-ray source with precise X-ray focusing optics, achieving detection sensitivities in the parts-per-billion (attogram) regime. 